\title{
Complexity Comparison for Drinkers' and Normal People's EEG Using Wavelet Entropy
}

\author{
Jiufu Liu ${ }^{1, *}$, Lei Gao ${ }^{2}$, Zaihong Zhou ${ }^{3, *}$, Haiyang Liu ${ }^{\mathrm{d}}$, Zhengqian Wang ${ }^{1}$, \\ Wenyuan Liu ${ }^{1}$ and Jianyong Zhou ${ }^{4}$ \\ ${ }^{1}$ College of Automation, Nanjing University of Aeronautics and Astronautics, \\ Nanjing 210016, China \\ ${ }^{2}$ Department of Information Management, Henan Information and Statistics \\ Vocational College, Zhengzhou 450008, China \\ ${ }^{3}$ School of Information Engineering, Guangdong Medical College, \\ Dongguan 523808, China \\ ${ }^{4}$ College of Electronic Science and Engineering, Southeast University, \\ Nanjing 210096, China \\ liujiufu2@126.com(JiufuLIU), sqzhou2012@126.com(Zaihong ZHOU)
}

\begin{abstract}
This paper investigates the influence of alcohol on brain complexity. Considering electro-encephalogram (EEG) has the nonlinear dynamics characteristic of time-varying and non-stationary, we introduce the wavelet entropy (WE) analysis. We denoise EEG signal by using wavelet decomposition, then calculate the wavelet entropy of the denoised signal and analyze the nonlinear complexity. In 64 conductive poles experiments and in different stimulus experiments for FP2 electrode's EEG, the drinkers' EEG wavelet entropy is greater than normal people's. The wavelet entropy of every conductive pole of drinkers' or normal persons' is inconformity.
\end{abstract}

Keywords: EEG; wavelet transform; wavelet entropy; complexity

\section{Introduction}

Drunk driving is the major reason for traffic accidents. The regularity of drinkers' brain function change is an interesting topic. Generally, dizziness, tinnitus and show response especially for emergency, are typical symptoms after drinking. Brain is composed of a huge amount of nerve cells and each nerve cell connects to other nerve cells, making brain a complex non-linear system. Complexity can reflect the regularity of dynamic systems. The behaviour of various systems is different, and thus the regularity of the behaviour from these systems is also different. Complexity is capable of describing these differences and then further discriminating these systems.

Electroencephalogram (EEG) is a non-invasive, low-cost and effective technique for examining electrical activity of the brain and diagnosing brain diseases in clinical setting [1]. EEG is a type of non-stationary time series signal. It's hard to analyze EEG by linear method, such as time domain analysis and frequency domain analysis because of the noregularity caused by nonlinear and non-stationary factors. Therefore non-linear analysis methods could better facilitate opening out the characteristics and mechanisms of EEG [2] [15].

With the rapid development of non-linearity theory, complexity analysis is becoming a popular field for studying nonlinear dynamics of EEG time series. Although different methods have provided indirect evidence for synchronization EEG processes [3, 8], a tool for a quantitative evaluation of the complex EEG signal synchronization and its temporal dynamics is still lacking. In information theory, 'entropy' represents the irregularity of 
systems, and many complexity concepts are related to entropy. Entropy is a concept handling predictability and randomness, with higher values of entropy always related to less system order and larger randomness [4]. Approximate entropy (ApEn) was first put forward by Pincus et al., [5]. In [6], an ApEn-based epileptic EEG detection system using artificial neural networks was studied. These methods are based on information theory, such as permutation entropy (PE), ApEn, and other ones based on chaos theory. SampEn was an improved algorithm based on approximate entropy (Richman et al., 2000) [7].

$\mathrm{PE}$ and ApEn are better in distinguishing the EEG between drinkers and the control, but they can't be used for on-line analysis due to too much time-consuming. ApEn's counting process adopts Heaviside function, which is very sensitive to the threshold value and phase space dimension, and vulnerable to noise interference. It lacks relative consistency and the result shows much dependence on data length. SampEn displays relative consistency and less dependence on data length. Nevertheless, the similarity definition of vectors in SampEn is based on Heaviside function as in ApEn. Due to the inherent flaws of Heaviside function, problems still exist in the validity of the entropy definition, especially when small parameters are involved. To overcome these limitations, Wavelet entropy (WE) [13] (Osvaldo A. Rosso et al., 2001), a new nonlinear dynamic analysis method, can be used for analyzing the short time signal. WE algorithm needn't consider any parameters during the process of calculation. It can reduce the influence of noise, reflect the signal's confusion degree of frequency components and provide the dynamics characteristics. And it is simple and possesses both time-frequency limitations and robustness.

In this paper, we investigate the influence of alcohol on brain complexity based on wavelet entropy. The work is organized as follows. Section 2 introduces wavelet entropy method. Section 3 WE performances to the nonlinear signals are discussed. In Section 4, by calculating the wavelet entropy of drinkers' and normal people's EEG signal, we analyze the complexity of drinkers' and normal people's EEG signal. Finally, Section 5 draws the conclusions.

\section{Wavelet Entropy}

\subsection{Wavelet Transform}

Wavelet analysis $[9,10-12,14]$ is a signal processing method, which relies on the introduction of an appropriate basis and a characterization of the signal by the distribution of amplitude in the basis. If the wavelet is required to form a proper orthogonal basis, it has the advantage that an arbitrary function can be uniquely decomposed and the decomposition can be inverted (Mallat, 1989). The wavelet is a smooth and quickly vanishing oscillating function with good localization in both frequency and time. A wavelet family $\psi_{a, b}(t)$ is the set of elementary functions generated by dilations and translations of a unique admissible mother wavelet $\psi(t)$ :

$$
\psi_{a, b}(t)=|a|^{-1 / 2} \psi\left(\frac{t-b}{a}\right), a \in R, a \neq 0, b \in R
$$

Where $\mathrm{a}, \mathrm{b}$ are the scale and translation parameters, respectively, and $\mathrm{t}$ is time. As the scale parameter a increases, the wavelet becomes wider. Thus, one has a unique analytic pattern and its replications at different scales and with variable time localization.

The continuous wavelet transform of a signal $S(t) \in L^{2}(R)$ (the space of real square summable functions) is defined as the correlation between the function $S(t)$ with the family wavelet $\psi_{a, b}(t)$ for each a and $\mathbf{b}$ : 


$$
\left(W_{\psi} S\right)(a, b)=|a|^{-1 / 2} \int_{-\infty}^{+\infty} f(t) \psi\left(\frac{t-b}{a}\right) d t=\left\langle S, \psi_{a, b}\right\rangle
$$

For a special election of the mother wavelet function $\psi(t)$ and for the discrete set of parameters, $a_{j}=2^{-j}$ and $b_{j, k}=2^{-j} k$ with $j, k \in Z$ (the set of integers) the family

$$
\psi_{j, k}(t)=2^{\frac{j}{2}} \psi\left(2^{j} t-k\right) \quad j, k \in Z
$$

constitutes an orthonormal basis of the Hilbert space $L^{2}(R)$ consisting of finite-energy signals. The correlated decimated discrete wavelet transform provides a non-redundant representation of the signal and its values constitute the coefficients in a wavelet series. These wavelet coefficients provide full information in a simple way and a direct estimation of local energies at different scales. More-over, the information can be organized in a hierarchical scheme of nested subspaces called multi-resolution analysis in $L^{2}(R)$. In the present work, orthogonal cubic spline functions are employed as mother wavelets. Among several alternatives, cubic spline functions are in a suitable proportion with smoothness and numerical advantages and they have become a recommended tool for representing natural signals.

In the following, the signal is assumed to be given by the sampled values $S=\left\{s_{0}(n), n=1, \cdots, M\right\}$, corresponding to a uniform time grid with sampling time $t_{s}$. For simplicity the sampling rate is taken as $t_{s}=1$. If the decomposition is carried out over all resolutions levels, $\mathrm{N}=\log _{2} \mathrm{M}$, the wavelet expansion will be:

$$
S(t)=\sum_{j=-N}^{-1} \sum_{k \in Z} C_{j}(k) \psi_{j, k}(t)=\sum_{j=-N}^{-1} \gamma_{j}(t)
$$

where wavelet coefficients $C_{j}(k)$ can be interpreted as local residual errors between successive signal approximations at scales $j$ and $j+1$, while $\gamma_{j}(t)$ is the residual signal at scale $j$. It contains information of the signal $S(t)$ corresponding to frequencies $2^{j-1} \omega_{s} \leq|\omega| \leq 2^{j} \omega_{s}$.

\subsection{Wavelet Energy}

Since the family $\left\{\psi_{j, k}(t)\right\}$ is an orthonormal basic for $L^{2}(R)$, the concept of energy is linked with the usual notions derived from Fourier theory. Then, wavelet coefficient are given by $C_{j}(k)=\left\langle S, \psi_{j, k}(t)\right\rangle$, and the energy of a signal at each scale $j=-1,-2, \cdots,-N$, will be

$$
E_{j}=\left\|\gamma_{j}\right\|^{2} \sum_{k}\left|C_{j}(k)\right|^{2}
$$

The energy at each sampled time $\mathrm{k}$ will be

$$
E(k)=\sum_{j=-N}^{-1}\left|C_{j}(k)\right|^{2}
$$

In consequence, the total energy can be obtained by

$$
E_{\text {tot }}=\|S\|^{2}=\sum_{j<0} \sum_{k}\left|C_{j}(k)\right|^{2}=\sum_{j<0} E_{j}
$$


For the $\mathrm{j}^{\text {th }}$ scale, the wavelet energy ratio is considered as a normalized value

$$
p_{j}=\frac{E_{j}}{E_{t o t}}
$$

The wavelet energy ratio vector $\left\{p_{j}\right\}$ represents energy distribution in a time-scale, which gives a suitable tool for detecting and characterizing singular features of a signal in time-frequency domain. Clearly, $\sum_{j=-1}^{-N} p_{j}=1$.

\subsection{Wavelet Entropy}

Entropy gives a useful criterion for analyzing and comparing a probability distribution. It provides a measure of information of any distribution. According to the entropy theory and wavelet energy ratio defined above, wavelet entropy is defined as

$$
S_{W T}=S_{W T}(p)=-\sum_{j<0} p_{j} \cdot \ln \left[p_{j}\right]
$$

To some extent, wavelet entropy can represent the degree of order/disorder of the signal, so it can provide useful information about the underlying dynamical process associated with measured signals. A signal generated by a totally random process can be taken as representing a very disordered behavior. This kind of signal will have a wavelet representation with significant contributions from all frequency bands. In addition, it is expected that all contributions will be of the same order. Consequently, the relative wavelet energy will be almost equal for all resolution levels and the wavelet entropy will take the maximum value.

\section{WE Performances to Nonlinear Signals}

In order to study the wavelet entropy's performance of mutation detection and sequence complexity measuring about the nonlinear time series, we construct the following ideal nonlinear time series.

$$
y_{n+1}=u y_{n}\left(1-y_{n}\right), y \in[0,1]
$$

In the Eq.11, $y_{0}=0.8, u=3.8$. The length of the ideal nonlinear time series still take 2000 , and the curve is shown in Figure 1(a). When the sequence at $\mathrm{t}=1001$, the evolution of the system becomes a random process, so that the complexity of the system significantly increases, and the predictability becomes smaller.

Similar to the method of introducing noise into ideal linear time series, we introduce the Gaussian white noise into nonlinear ideal time series in the foundation of Eq.11. The Gaussian white noise with noise ratio for $2 \mathrm{db}$, and the amplitude for 0.2. The noised nonlinear time series signal curve is shown in Figure 1(b).

Figure 2 is the entropy curve of ideal nonlinear time series. In $1 \leq t \leq 1000$ two kinds of entropy values are obvious smaller than that in $1000<t \leq 2000$. In Figure 2 (a), when the scale of the sliding window is smaller, with the movement of the sliding window, ApEn curve is unstable and changing larger, because the data is so short that the approximate entropy values are not enough robust. With the length of the sliding window increasing, in Figure 2 (b) the ApEn curve becomes more stable, and it can reflect out the sudden change of the dynamical structure of the system. In Figure 2 (a) and (b), from $t=800$ to $t$ $=1001$, the curve reduces first and then increases significantly, we can roughly determine the mutation starts near $t=1000$. Because the curve fluctuates frequently in the area, there are some difficulties to locate the mutation point. 


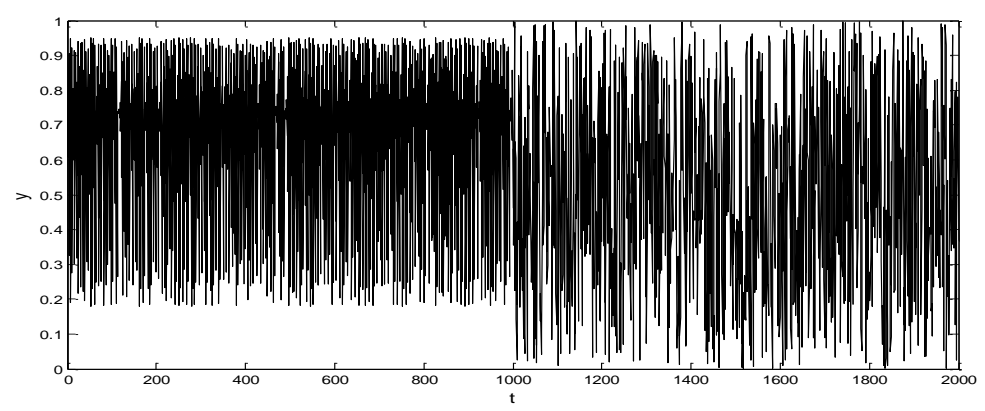

(a)

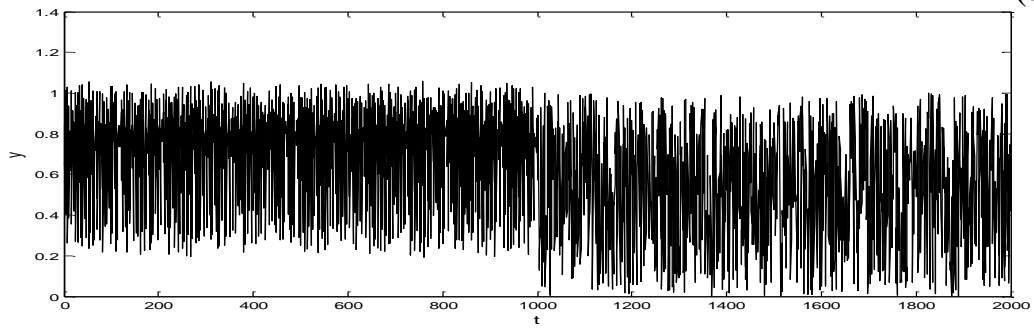

(b)

Figure 1. Nonlinear Time Series (a) Ideal Nonlinear Time Series, (b)Nonlinear Time Series with Gaussian White Noise
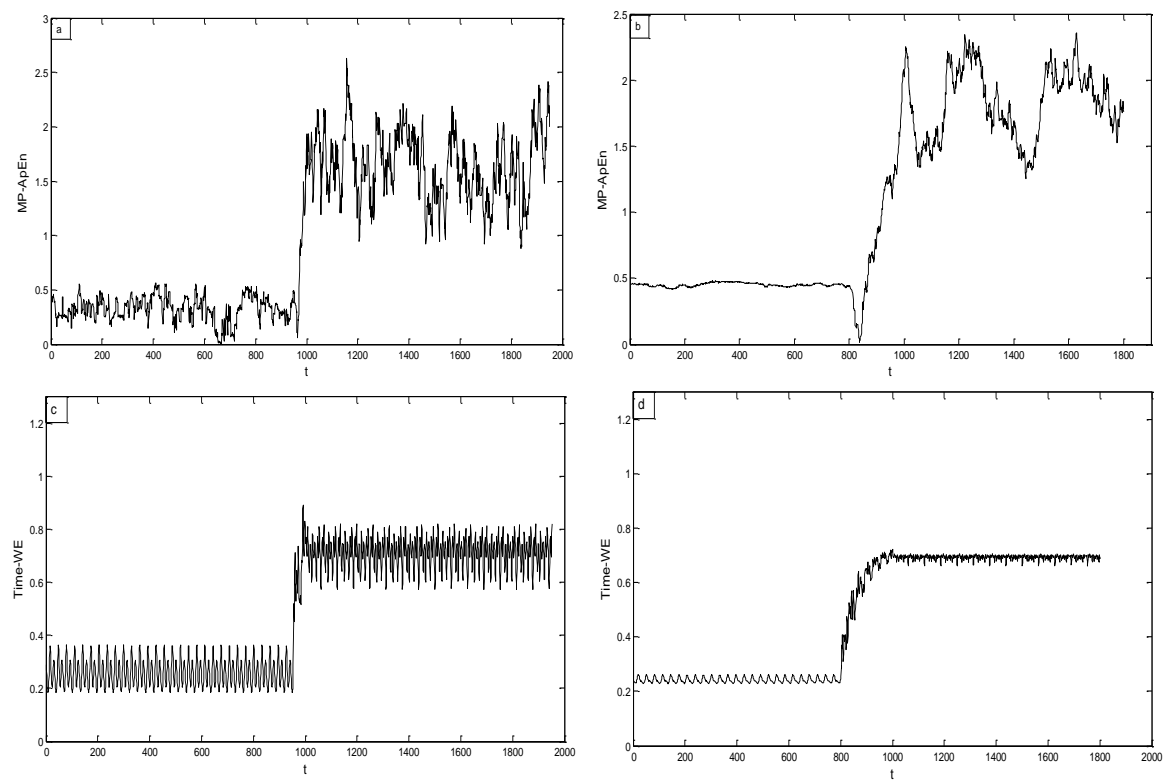

Figure 2. Entropy Curves of Ideal Nonlinear Time Series. MP-ApEn Curve: (a) Sliding Window Length $L=50$, step 1; (b) $L=200$. Time-WE Curve: (c) Time Evolution Window Length L=50, Step 1; (d) $L=200$

However, according to the Time-WE curves fluctuating in Figure 2 (c) and (d), we see that with the length of time evolution window changing, the fluctuation of the curve is relatively small, and when the length of time evolution window $\mathrm{L}=50$ and $\mathrm{L}=200$, the entropy curves variation tendency is similar. Time-WE is nearly independent of the length of the time window, WE can accurately reflect the structural change of the sequence. From Time-WE in Figure 2 (c) and (d), the wavelet entropy can accurate quantitative measure the complexity of the nonlinear time series, which is sensitive to the dynamic complexity changing for the nonlinear time series. The mutation point of the nonlinear time series is near $\mathrm{t}=1000$.

In reality, most of signals are nonlinear signal, which blend various noises in different degree. To study the performance of wavelet entropy for measuring the complexity of 
nonlinear time series with Gaussian white noise, we calculate MP-ApEn and Time-WE of the noised nonlinear time series. The MP-ApEn and Time-WE curves are shown in Figure 3. By analyzing (a) and (b) in Figure 3 and Figure 2, especially the first part of the curves, ApEn values are all under 0.5 until near $t=800$ in Figure 2, and on the contrary ApEn values are all whereon 0.5 in Figure 3, we can learn that because the original signal and noise signal are no longer independent, the result of noise for ApEn is making sequences entropy increase, namely, the confusion degree of series is more greater. However, in Figure 2(c) (d) and Figure 3(c) (d), the initial value, transition value and the overall trend of the WE value curve are almost no change. This can explain that the wavelet transform reduce the effect of noise during the calculation of WE, which makes calculation of WE be more accurate, and WE can reflect the chaos degree of the nonlinear time series.
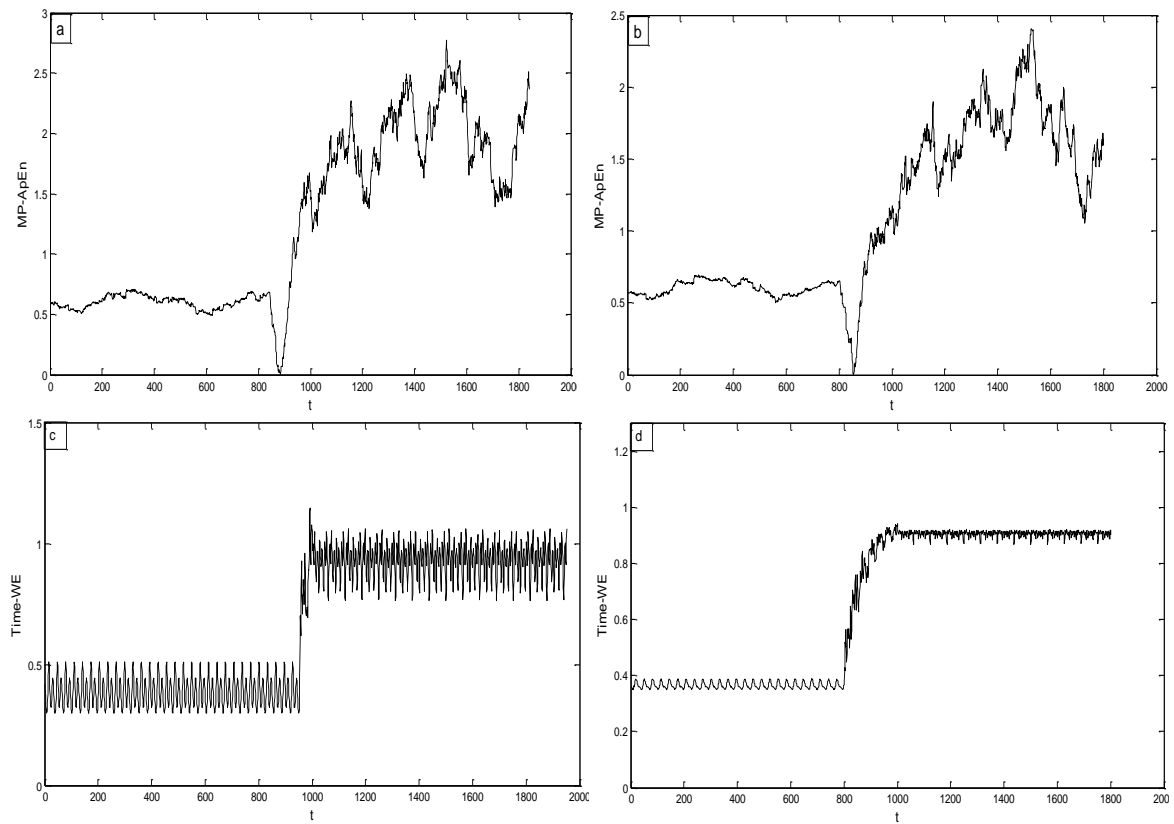

Figure 3. Curves of Nonlinear Noised Time Series in Mutation Detection. MP-ApEn Curve: (a) Sliding Window Length $L=50$, step 1; (b) $L=200$. TimeWE Curve: (c) Time Evolution Window Length $L=50$, Step 1; (d) $L=200$

\section{Application Examples}

\subsection{Experiment Data}

The experimental data were taken from a public EEG database. The experiments were performed on 122 subjects. The tested people were made experiments 120 times respectively (Zhu Guohun et al., 2011) [16]. In the experiment, the tested people's heads were placed with 64 conductive poles, the sampling frequency was $256 \mathrm{~Hz}$ and recording data period was 1 second in every experiment. Because the data of EEG in data concentration is incomplete, some experiment data are not in the database, therefore, with the requirements of examples analysis and in order to ensure the comparability of analysis results, 30 drinkers' and 30 normal persons' EEG completely data were selected at random in the dataset, as two data sets of this research analysis. Firstly, we calculate the sampling data of 64 conductive poles that were got in single at the 40th stimulation experiment, and analyze the results. Then, under the condition of three kinds of stimulation experiments, we calculate and analyze EEG data of FP2 conductive pole, which belonged to drinkers numbered co2a0000364 and normal people numbered co2c0000337 in the same 10 times stimulation experiments. 


\subsection{Experiment Results and Analysis}

\subsubsection{The Whole Influence of Alcohol on the Brain}

We calculate all 64 electrodes' wavelet entropy to get the average value, the EEG data selected from the 40 times experiment about 60 tested people, and draw drinkers' and normal persons' average wavelet entropy curve of 64 conductive poles. As shown in Figure 4. Among the 60 tested people, there were 30 drinkers and 30 normal persons.

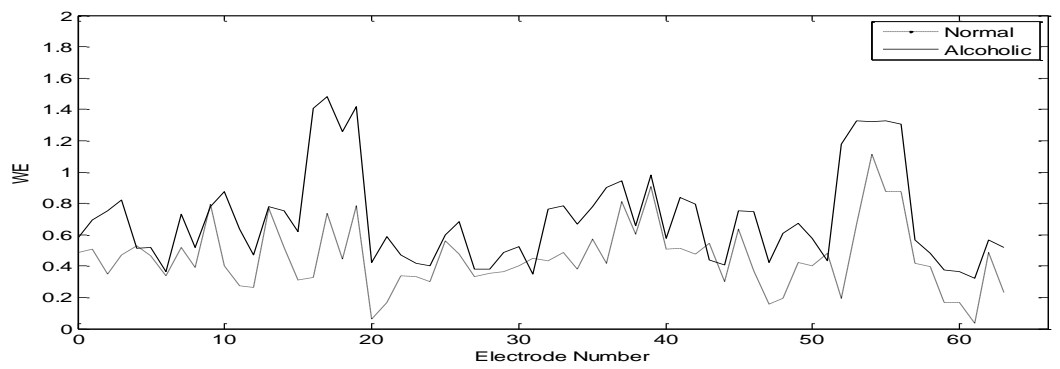

\section{Figure 4. WE Curves of 64 Conductive Poles of Drinkers' and Normal People's}

In Figure 4, including 64 conductive poles, the drinkers' EEG wavelet entropy is widely greater than normal people's. The wavelet entropy of every conductive pole of drinkers' or normal persons' is inconformity, and the degree of EEG wavelet entropy between drinkers and normal persons is also different at the same conductive pole, which explains the distribution of EEG signal is not uniform in head.

Compared with normal brain, the drinkers' will be with highly complexity and neuronal activity is increased because of stimulation of alcohol. All results can properly reflect the dynamics nature and changes of brain signals. For the changes between drinker's and normal people's EEG wavelet entropy at the same electrode point, from Figure 4, at CP1, CP5 and CP6 electrode points, the increasing trend of WE is obvious larger, which means these parts are more sensitive to alcohol. This provides a more reliable and keen way to determine person whether has drunk.

The method based on WE to analyze the complexity of EEG signal, can be made a contribution to detecting drunken driving and helping hospitals with alcoholism.

\subsubsection{FP2 Electrode's EEG Analysis in Different Stimulus Experiment}

In order to analyze WE's obvious differences of two groups of EEG data on the same electrode, and make further analysis in the impact of alcohol on person 's health, we choose alcoholic numbered co2a0000364 and normal people numbered co2c0000337 to carry on the same experiments 10 times in the condition of three kinds of external stimulation experiments. Each subject was exposed to either a single stimulus $\left(\mathrm{S}_{1}\right)$ or two stimuli $\left(S_{1}\right.$ and $\left.S_{2}\right)$ which were pictures of objects chosen from the 1980 Snodgrass and Vanderwart picture set. When two stimuli were shown, they were presented in either a matched condition $\left(S_{m}\right)$ where $S_{1}$ was identical to $S_{2}$ or in a non-matched condition $\left(S_{n}\right)$ where $S_{1}$ differed from $S_{2}$.

(1) While in the condition of a single stimuli $\left(S_{1}\right)$ experiment, we can get the FP2 electrode's EEG data form the experiment. FP2 electrodes were located at the upper part of the eyes. Calculating WE of drinker and normal people at FP2 electrode, we get the wavelet entropy in Figure 5(a). As in Figure 8(a), under the condition of a single stimulus experiment, the WE of drinkers' EEG data on FP2 electrode is markedly greater than normal people's. We can also find that entropy curves become more and more stable with more experiments. 
(2) While in the experiments of continuous matched stimuli condition, we can get the FP2 electrode's EEG data from the experiments. By calculating WE of drinkers' and normal people's at FP2 electrode in every experiment, we get the wavelet entropy in Figure 5(b). From Figure 5(b), the WE of drinkers' EEG data on FP2 electrode is still greater than normal people's under the experiments of matched stimuli condition and the normal people's EEG entropy becomes smaller and smaller. In other words, the brain's memory consciousness of normal people's is enhanced, and more easily form a condition reflex, etc.
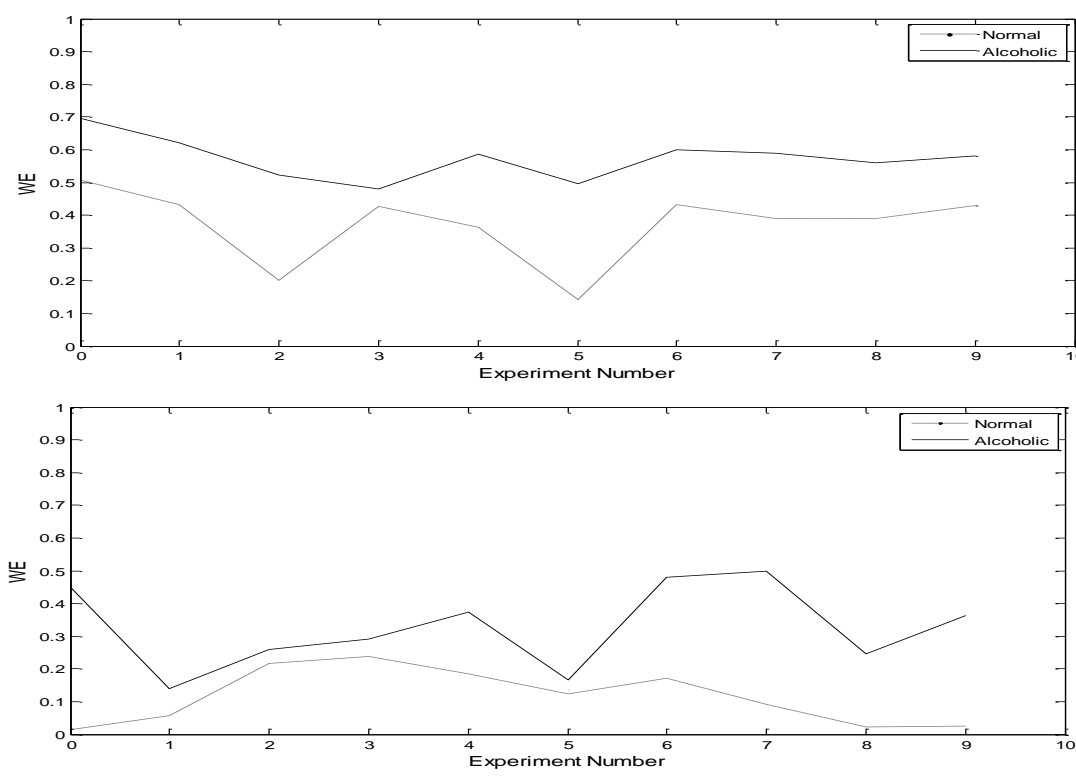

(a)

(b)

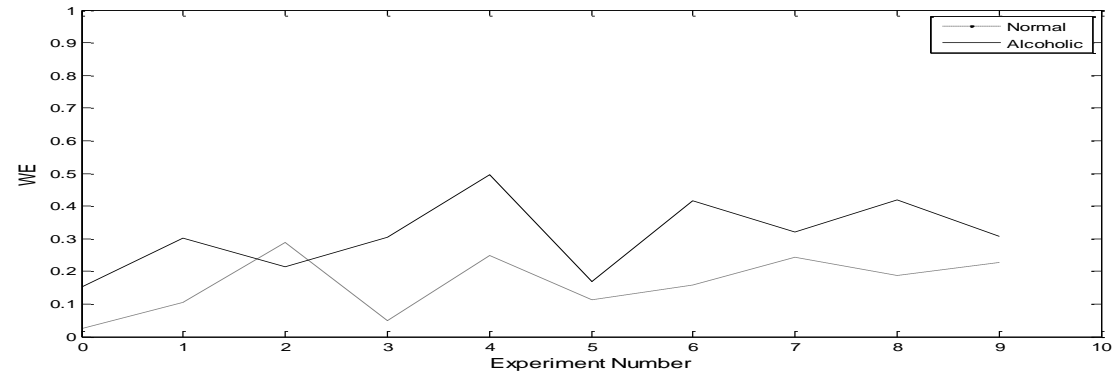

(c)

\section{Figure 5. WE Curve of FP2 Electrode of Drinkers' and Normal People's (a) Under a Single Stimulation Experiment, (b)Under a Continuous Matched Stimuli,(c) Under a Continuous Non-Matched Stimuli}

(3) While in experiment of continuous non-matched stimuli condition, we calculate WE of alcoholic and normal people at FP2 electrode in every experiment, and get the wavelet entropy in Figure 5(c). In the Figure 8(c), under the condition of non-matched stimuli, the WE of drinkers' EEG data on FP2 electrode is mostly greater than normal people's, but the complexity of normal people's brain is larger in the third experiment in Figure 8(c). With the experiment carried out, the WE wave of alcoholics fluctuates more intensely. However, the normal people's wave is gradually leveling off. Comparing with drinkers, the normal people's control and cognitive ability is more sustained to outside different stimulus, the normal people's brain can make accurate response more quickly. 


\section{Conclusion}

In this paper, we introduced the wavelet entropy to analyze and measure the EEG complexity of drinkers' and normal people's.

(1) Wavelet decomposition can reduce the influences of noise. WE model dimension $m$ and threshold value $r$, have no influence on the result. We can accurately measure the chaos of nonlinear time series.

(2) Comparing and analyzing drinkers' (or alcoholics') and normal people's EEG, the WE of drinkers' is widely larger than that of normal people's. In other words, the complexity of drinkers' brains is higher than that of normal people's. This makes a contribution to studying the states and complexity of drinkers' brains.

Considering that drinkers' intoxication levels and sensitive degree for alcohol are all different, we will introduce fuzzy analysis method, such as fuzzy entropy, based on quantitative WE analysis in future works. We will do systematic fuzzy classification of the drunken degree and alcohol sensitive degree of drinkers', and reveal the changing rules of drinkers' EEG complexity with more details.

\section{Acknowledgements}

The research has been supported by the Nanjing University of Aeronautics and Astronautics basic scientific research Funds (NS2010069).

\section{References}

[1] C. J. Stam, J. P. Pijn and P. Suffczynski, "Dynamics of the human alpha rhythm evidence for nonlinearity", Clin Neurophysiol, vol. 110, no. 10, (1999), pp. 1801-1813.

[2] D. Duke and W. Pritchard, "Measuring chaos in the human brain", Singapore: World Scientific, (1991).

[3] H. D. I. Abarbanel, "Analysis of observed chaotic data", New York: Springer, (1996).

[4] N. Kannathal, L. M. Choo and U. R. Acharya, "Entropies for detection of epilepsy in EEG", Comput Methods Prog. Biomed, vol. 80 (2005), pp. 187-194.

[5] S. Pincus, "Approximate entropy (ApEn) as a complexity measure", Chaos, vol. 5, no. 1, (1995), pp. 110-117.

[6] V. Srinivasan, C. Eswaran and N. Sriaam, "Approximate entropy-based epileptic EEG detection using artificial neural networks", IEEE Trans Inf Technol Biomed, vol. 11, no. 3, pp. 288-295.

[7] J. S. Richman and J. R. Moorman, "Physiological time-series analysis using approximate and sample entropy”, Am J Physiol Heart Circ Physiol., vol. 278, no. 6, (2000), pp. H2039-2049.

[8] J. M. Guo and K. Tsair, "Alter ation detection and recovery for medical and surveillance systems", ICIC Inter Conf, vol. 9, no. 4, (2013), pp. 1389-1408.

[9] O. Hasan, "Automatic detection of epileptic seizures in EEG using discrete wavelet transform and approximate entropy", Expert Systems with Applications, vol. 36, no. 2, (2009), pp. 2027-2036.

[10] M. Kohei and Y. Tomonari, "EEG Analysis During Sleep by Using Morphological Local Pattern Spectrum", ICIC Express Letters, vol. 7, no. 5, pp. 1469-1474.

[11] Z. Li and Y. Wang, "Classification of Sound Types in Border Monitoring System Based on Wavelet Transform”, ICIC Express Letters, Part B: Applications, vol. 3, no. 1, (2012), pp. 127-132.

[12] S. G. Mallat, "A theory for multi resolution signal decomposition: The wavelet representation", IEEE Trans Pattern Anal Mach Intelligence, vol. 11, no. 7, (1989), pp. 674-693.

[13] A. R. Osvaldo and B. Susana, "Wavelet entropy: a new tool for analysis of short duration brain electrical signals", Journal of Neuroscience Methods, vol. 105, no. 1, (2001), pp. 65-75.

[14] T. Qian and Y. Noriyoshi, "An Approach Based on Wavelet Analysis and Hidden Markov Models for Behavior Understanding", ICIC Express Letters, Part B: Applications, vol. 3, no. 6, (2012), pp. 16451650.

[15] Y. Fang and X. Zheng, "Feature Extraction of Time-Amplitude-Frequency Analysis for Classifying Single EEG", Journal of Fiber Bioengineering \& Informatics, vol. 7, no. 2, (2014), pp. 261-271.

[16] G. H. Zhu, Y. Li and P. Wen, "Evaluating function connectivity in alcoholics based on maximal weight matching", Journal of Advanced Computational Intelligence and Intelligent Informatics, vol. 15, no. 9, (2011), pp. 1221-1227. 
International Journal of Hybrid Information Technology

Vol.8, No.8 (2015) 